\title{
Oculopharyngeal Muscular Dystrophy
}

National Cancer Institute

\section{Source}

National Cancer Institute. Oculopharyngeal Muscular Dystrophy. NCI Thesaurus. Code C84942.

An autosomal dominant disorder caused by mutations in the PABPN1 gene, encoding polyadenylate-binding protein 2 . The condition is characterized by progressive ptosis, dysphagia and weakness of the muscles of the face, neck, and extraocular muscles. 